\title{
Excitation of field line resonances by sources outside the magnetosphere
}

\author{
A. D. M. Walker \\ School of Physics, University of KwaZulu-Natal, Durban 4041, South Africa \\ Received: 14 June 2005 - Revised: 27 September 2005 - Accepted: 28 September 2005 - Published: 30 November 2005
}

\begin{abstract}
Field line resonances are thought to be excited by sources either at the magnetopause or outside it. Recent observations suggest that they may be associated with coherent oscillations or pressure pulses in the solar wind. In either case the excitation mechanism can be understood by considering the incidence of a harmonic wave on the magnetopause from outside the magnetosphere. Calculations are performed in a plane stratified model that consists of (i) a magnetosheath region streaming tailward at uniform velocity (ii) a sharp boundary representing the magnetopause, (iii) a magnetosphere region in which the Alfvén speed increases monotonically with distance from the magnetopause. The structure implies the existence of a propagating region within the magnetopause bounded by a reflection level or turning point. Beyond this is a region in which waves are evanescent and a resonance level. The reflection and transmission of harmonic waves incident from the magnetosheath is considered in this model. It is shown that, in most cases, because of the mismatch between the magnetosphere and the magnetopause, the wave is reflected from the magnetopause with little penetration. At critical frequencies corresponding to the natural frequencies of the cavity formed between the magnetopause and turning point the signal excites the cavity and may leak evanescently to the resonance. The calculation includes the effect of the counter-streaming magnetosheath and magnetosphere plasmas on the wave. This can lead to amplification or attenuation. The nature of the processes that lead to transmission of the wave from magnetosheath to resonance are considered by synthesising the signal from plane wave spectra. A number of mechanisms for exciting cavity modes are reviewed and the relationship of the calculations to these mechanisms are discussed. Observations needed to discriminate between the mechanisms are specified.
\end{abstract}

Keywords. Magnetospheric physics (MHD waves and instabilities; Solar wind-magnetosphere interactions) - Space plasma physics (Kinetic and MHD theory)

Correspondence to: A. D. M. Walker

(walker@ukzn.ac.za)

\section{Introduction}

The source of discrete field line resonances in the magnetosphere is still a matter for debate. Early observations of the structure of field line resonances (Walker et al., 1979) were over a relatively small range of latitudes and showed a single field line resonance. The most plausible source at that time was a Kelvin-Helmholtz instability on the magnetopause, as first envisaged by Dungey (1954) and Southwood (1968). The very well-defined frequency of the resonance was difficult to explain in this way and this led Kivelson and Southwood $(1985,1986)$ to suggest that the discrete frequencies were the eigenfrequencies of cavity modes just inside the magnetopause, coupled to the field line resonances. This suggestion received strong experimental support with the HF radar observations of Ruohoniemi et al. (1991) who observed several simultaneous field line resonances at different latitudes and frequencies. Such a discrete frequency spectrum would be difficult to explain as the result of a simple KelvinHelmholtz instability. It was later suggested (Walker et al., 1992; Samson et al., 1992) that the cavity behaved more like a waveguide and substantial attention has been paid to the study of modes in such a waveguide and their excitation (Wright, 1994; Wright and Rickard, 1995a,b). Recently there have been suggestions that the source of the field line resonance may be monochromatic MHD waves in the in the solar wind (Stephenson and Walker, 2002; Kepko et al., 2002), impinging on the magnetopause.

The excitation of the magnetospheric waveguide has been the subject of theoretical study by Mann et al. (1999) who allowed for the effect of a leaky boundary and magnetosheath flow, but not for coupling to the resonance. They considered the source to be at the magnetopause and thus were able to show how the Kelvin-Helmholtz instability could excite waveguide modes. An important aspect of their treatment was the inclusion of over reflection effects which could lead to the modes being amplified at the magnetopause. An alternative approach was that of Walker (1998) who considered the excitation of the waveguide by a wave incident on the magnetopause from outside the magnetosheath, but did not 
allow for coupling to the resonance. Magnetosheath motion was implicit in the treatment but not included in the computations.

The purpose of this paper is to extend the results of Walker (1998) to include the effects of coupling to the resonance and magnetosheath motion in the calculations. The focus is on waves incident from outside the magnetosphere. The work is therefore complementary to that of Mann et al. (1999) who were concerned with excitation at the boundary layer. The approach is applicable to any disturbance incident from outside.

In Sect. 2 we summarise the equations necessary to study the problem and then in Sects. 3 and 4 describe the results of computations of the nature of the signal. These computations show how the cavity is excited in the absence of a resonance and when the velocity of the magnetosheath is negligible. They are then generalised to show the effect of considering leakage of energy to the resonance and of a moving magnetosheath. Finally, in Sect. 5, the relative importance of various excitation mechanisms and the necessary experimental information that would be required to distinguish between them are discussed.

\section{Equations}

The plane stratified system described by Walker (1998) is illustrated in Fig. 1. The upper panel shows a schematic diagram of the equatorial plane of the magnetosphere. The magnetopause separates the magnetosphere from the magnetosheath plasma, which is streaming anti-sunward. A wave is incident from the magnetosheath. At the magnetopause it is partly reflected and partly transmitted. Inside the magnetopause it is propagated to the turning point where the increasing Alfvén speed leads to reflection. It is reflected back and forth between turning point and magnetopause. Beyond the turning point it leaks evanescently to excite the resonance.

The lower panel maps this process into a box model. The advantages and limitations of such models are discussed by Walker (1998). The magnetopause is represented by a tangential discontinuity at $x=0$ separating two magnetohydrodynamic media in relative motion. The $x$ axis is normal to the boundary and the properties of each medium depend only on the coordinate $x$. The turning point is at $x=x_{T}$ and the resonance at $x_{R}$. The variation is assumed sufficiently slow for the WKBJ approximation to be valid. The streaming velocity is everywhere parallel to the boundary. For the purposes of this paper medium 1 has a uniform streaming velocity in the $y$ direction and represents the magnetosheath. Medium 2 is at rest and represents the magnetosphere. The dependence of magnetic field, plasma density, and pressure vary with $x$ in a qualitatively similar way to their behaviour in the magnetosphere, although, in this simple illustrative box model, we do not attempt quantitative agreement.

We assume a magnetosonic wave is incident from medium 1 on the boundary and is partly reflected and partly transmitted. Transverse Alfvén waves cannot participate in the process since they are propagated parallel to the magnetic field which, for a tangential discontinuity, is parallel to the boundary. Depending on frequency the transmitted wave may be propagated in medium 2 or it may be evanescent. If it is propagated it encounters a turning point where it is reflected. If there is an accessible resonance beyond the turning point, part of the energy reaches the resonance by barrier penetration.

We also assume that, in medium 1 , and in medium 2 between the boundary and the turning point, the medium is sufficiently slowly varying that the wave is adequately described by the WKBJ solution. In medium 1 the WKBJ solution for the generalised pressure perturbation $\psi \equiv p+\mathbf{B} . \mathbf{b} / \mu_{0}$ is (Walker, 2005, §20.5)

$\psi=\frac{\mathcal{F}^{1 / 2}}{\mathcal{G}^{1 / 4}}\left\{\exp \left[i \int^{x} \sqrt{\mathcal{G}} d x\right]+R \exp \left[-i \int^{x} \sqrt{\mathcal{G}} d x\right]\right\}$,

where $R$ is a reflection coefficient to be determined. In medium 2 the WKBJ solutions are

$\psi=T \frac{\mathcal{F}^{1 / 2}}{\mathcal{G}^{1 / 4}}\left\{\exp \left[i \int^{x} \sqrt{\mathcal{G}} d x\right]+S \exp \left[-i \int^{x} \sqrt{\mathcal{G}} d x\right]\right\}$.

The two terms in the WKBJ solutions in each medium represent waves propagated in the positive and negative $x$ directions, respectively. The amplitude $T$ is a transmission coefficient representing the ratio of the amplitude of the signal in the guide to that of the incident wave. The quantity $S$ is the Stokes constant that allows the WKBJ solutions to be matched at the turning point.

When there is no coupling to the resonance the Stokes constant is

$S=-i \equiv e^{-i \pi / 2}$.

Since $|S|$ represents the ratio of the amplitudes of the waves incident on and reflected from the turning point, and pha $(S)$ their relative phase, the amplitudes of incident and reflected waves are the same, and there is total reflection with a phase change of $-\pi / 2$ on reflection.

If the resonance is close enough to the turning point for coupling to be important then $S$ must be found by other means as described by Walker (2000b).

The WKBJ solutions are not particularly realistic. However, the purpose of this paper is not to provide quantitative results but insight into the nature of the process that allows waves to be captured by the waveguide region and transmitted to the resonance. The WKBJ solutions allow the disturbance to be visualised as incident and reflected waves that can be easily identified with the characteristic waves in a uniform medium. WKBJ solutions are accurate when the medium is sufficiently slowly varying. Even when the conditions for their accuracy are not strictly met they may still give a good representation of the phase of the wave, although they are not such a good approximation for the amplitude. A detailed discussion of the accuracy of WKBJ solutions in a slowly varying medium and near the turning point and resonance is given by Walker $(2005, \S 15.4, \S 19.2)$. 


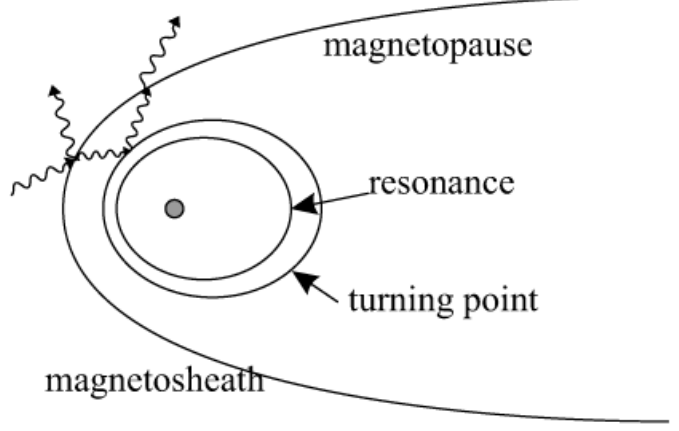

(a)

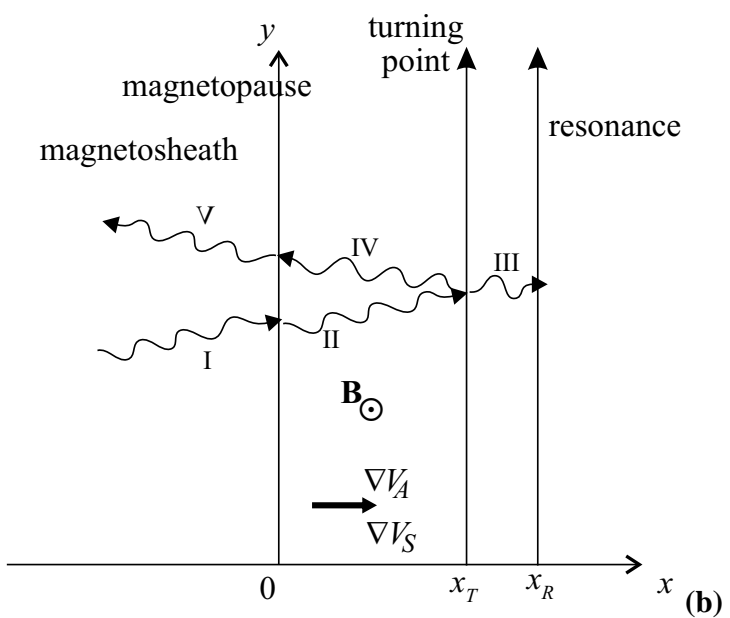

Fig. 1. Box model of the magnetospheric waveguide or cavity. (a) Equatorial plane of magnetosphere (b) Mapping to a box model (see text for explanation)

The nature of the reflection and transmission process and the calculation of $R$ and $T$ has been discussed by Walker (1998) for the case where there is no coupling to the resonance and $S=-i$. The treatment allows for relative motion of magnetosheath and magnetosphere, although the numerical computations were only carried out for the case where the media are at rest. This treatment is easily generalised to the case where there is coupling to the resonance. It is only necessary to replace the Stokes constant $-i$ in the equations of Walker (2002) by the appropriate constant $S$.

This gives formulae for the reflection and transmission coefficients $R$ and $T$ :

$R=\frac{\sin \Theta+i \chi \cos \Theta}{\sin \Theta-i \chi \cos \Theta}$

$T=\frac{-i e^{i \Theta}}{\sin \Theta-i \chi \cos \Theta}$

where

$$
\begin{aligned}
\Theta & =-\left\{\int_{0}^{x_{T}} k_{x} d x-\frac{1}{2} i \ln S\right\} \\
\chi & =\frac{\mathcal{F}\left(0^{-}\right)}{\mathcal{F}\left(0^{+}\right)} \sqrt{\frac{\mathcal{G}\left(0^{+}\right)}{\mathcal{G}\left(0^{-}\right)}}=\frac{\mathcal{F}\left(0^{-}\right)}{\mathcal{F}\left(0^{+}\right)} \frac{k_{x}\left(0^{+}\right)}{k_{x}\left(0^{-}\right)} \\
\mathcal{F} & =\rho_{0}\left\{\omega_{0}^{2}-\left(\mathbf{k} \cdot \mathbf{V}_{A}\right)^{2}\right\} \\
\mathcal{G} & =\frac{\omega_{0}^{4}}{\omega_{0}^{2}\left(V_{A}^{2}+V_{S}^{2}\right)-\left(\mathbf{k} \cdot \mathbf{V}_{A}\right)^{2} V_{S}^{2}}-k_{y}^{2}-k_{z}^{2} \\
\omega_{0} & =\omega-\mathbf{k} \cdot \mathbf{V}
\end{aligned}
$$

and where the branches of $k_{x}$ are chosen on the real $\Omega$ axis, so that:

- If $k_{x}$ is real it corresponds to a wave propagated in the $+x$ direction,

- If $k_{x}\left(0^{-}\right)$is imaginary it represents an evanescent wave decaying in the positive $x$ direction.
The formulae derived by Walker (1998) are retrieved by setting

$$
\frac{1}{2} i \ln S=\frac{\pi}{4}
$$

The quantity $\omega_{0}$ is the Doppler shifted frequency that must be used in the dispersion relation to take account of motion of the medium.

When the wave is not propagated, but is evanescent within the magnetosphere (Walker, 1998, Eqns. 40, 41)

$$
\begin{aligned}
& 1+R=T \\
& 1-R=\chi T .
\end{aligned}
$$

This leads to

$$
\begin{aligned}
R & =\frac{1-i \operatorname{sgn}\left(\omega_{0}\right)|\chi|}{1+i \operatorname{sgn}\left(\omega_{0}\right)|\chi|} \\
T & =\frac{2}{1+i \operatorname{sgn}\left(\omega_{0}\right)|\chi|}
\end{aligned}
$$

\subsection{Simple model}

In order to illustrate the use of the formulae for $R$ and $T$ we use a very simple model. The magnetic field in medium 1 (the magnetosheath) is assumed small enough to be neglected so that we can set $V_{A}=0$ in the magnetosheath. The streaming velocity in this medium is $\mathbf{V}$ in the $y$ direction. Within the magnetosphere $\beta$ is small, so that we can set $V_{S}=0$. The magnetic field $\mathbf{B}$ is in the $z$ direction. At the boundary pressure balance requires that

$P(0)=\frac{B^{2}(0)}{2 \mu_{0}}$.

Within the magnetosphere $V_{A}$ varies as

$V_{A}(r)=\frac{a L_{0}}{r} V_{A, 0}$ 
where

$r=a L_{0}-x$

and $L_{0}$ is the distance of the magnetopause from the centre of the Earth, measured in Earth radii. This linear variation of $V_{A}$ is much weaker than is realistic but it allows easy analytical representations of the phase integrals.

The quantities $\mathcal{F}$ and $\mathcal{G}$, given by (8) and (9), in the two regions 1 and 2 are then

$$
\begin{aligned}
& \mathcal{F}_{1}=\rho_{0,1}\left(\omega-k_{y} V\right)^{2} \\
& \mathcal{F}_{2}=\rho_{0,2}\left(\omega^{2}-k_{z}^{2} V_{A, 2}^{2}\right) \\
& \mathcal{G}_{1}=\frac{\left(\omega-k_{y} V\right)^{2}}{V_{S, 1}^{2}}-k_{y}^{2}-k_{z}^{2} \\
& \mathcal{G}_{2}=\frac{\omega^{2}}{V_{A, 2}^{2}}-k_{y}^{2}-k_{z}^{2}
\end{aligned}
$$

\subsection{Normalisation of the equations}

We can normalise the frequencies, velocities, and wave numbers in terms of $a L_{0}$, the distance of the magnetopause from the centre of the Earth, and $V_{A, 0}$, the Alfvén speed at the magnetopause. Let

$$
\begin{aligned}
& l=a L_{0} k_{z} \\
& m=a L_{0} k_{y} \\
& \Omega=\frac{\omega}{V_{A, 0} \sqrt{k_{y}^{2}+k_{z}^{2}}} \\
& U_{S}=V_{S} / V_{A, 0} \\
& U=V / V_{A, 0} \\
& \Omega_{0}=\Omega-\frac{m U}{\sqrt{l^{2}+m^{2}}}
\end{aligned}
$$

The condition for pressure balance (16) requires that

$$
U_{S}^{2}=\frac{\gamma}{2} \frac{\rho_{0}\left(0^{+}\right)}{\rho_{0}\left(0^{-}\right)}
$$

\subsection{Evaluation of $R$ and $T$}

The integral in the expression for $\Theta$ can be evaluated by elementary means giving

$\Theta=\frac{1}{2} i \ln S-\frac{\sqrt{l^{2}+m^{2}}}{2}\left\{\sqrt{\Omega^{2}-1}-\frac{\ln \left[\Omega+\sqrt{\Omega^{2}-1}\right]}{\Omega}\right\}$

and

$$
\chi=\frac{\rho_{0}\left(0^{-}\right)}{\rho_{0}\left(0^{+}\right)} \frac{\Omega_{0}^{2}}{\Omega^{2}-l^{2} /\left(l^{2}+m^{2}\right)} \sqrt{\frac{\Omega^{2}-1}{\Omega_{0}^{2} / U_{S}^{2}-1}}
$$

This allows us to plot the reflection and transmission coefficients as a function of normalised frequency $\Omega$.
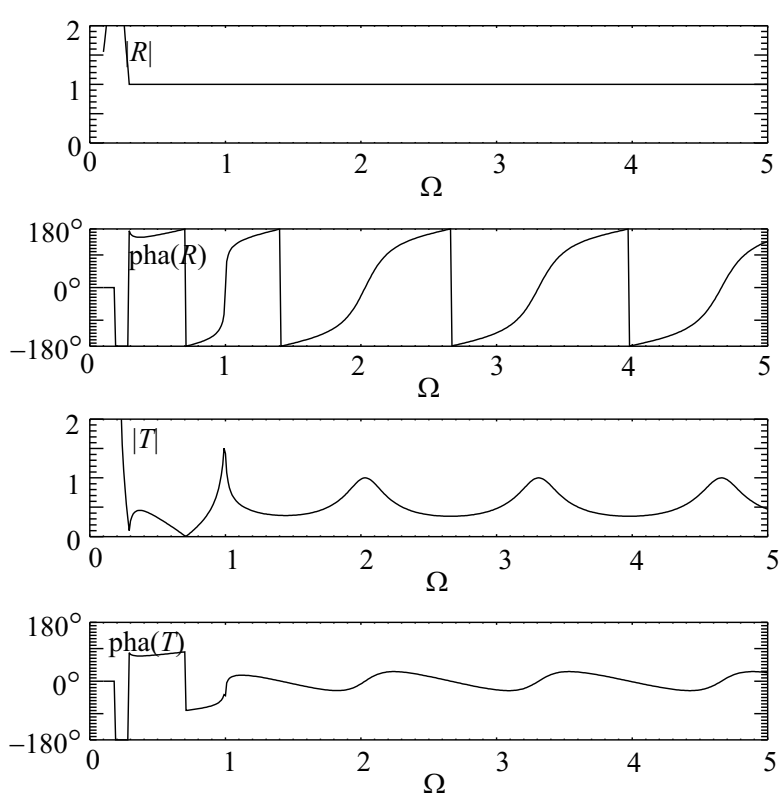

Fig. 2. Amplitude and phase of the reflection and transmission coefficients when excitation of the resonance is negligible. $\rho_{0,2}=0.1 \rho_{0,1}, k_{z}=3 / a L_{0}, k_{y}=3 / a L_{0}, V / V_{A, 0}=0$.

\section{Computations}

\subsection{Excitation of the cavity}

Walker (1998) calculated the values of $R$ and $T$ as functions of $\Theta$, with $\chi$ as a parameter, for the case where the magnetosheath velocity was negligible and coupling to the resonance was ignored. This calculation was independent of the model. Here, in Fig. 2 we show them for our model as functions of the normalised frequency $\Omega$. When $\Omega<1$ we use Eqs. (14) and (15) and when $\Omega>1$ we use Eqs. (4) and (5).

When $\Omega<1$ the wave in medium 2 is evanescent. There is no turning point and no possibility of cavity resonance.

At $\Omega=U_{S}$ there is a zero of $k_{x}$ in medium 1. For these parameters it occurs at $\Omega=0.29$. For $0.29<\Omega<1$ there is total reflection of the wave, and the magnitude of the reflection coefficient is unity. For smaller frequencies than this there is no propagation in medium 1. It might be supposed that this region is not of physical interest since the waves are evanescent there and cannot carry energy into the region. There is the possibility, however, that there may be a boundary at some negative value of $x$ that is not much more than an $e$ folding distance away. In this case energy may enter through evanescent barrier penetration. Above this frequency the incident waves are propagated.

If we examine the amplitude of the reflection coefficient in the region $\Omega<0.29$ we observe that it has a singularity near $\Omega=0.2$ where $\chi=-1$. The physical interpretation of an infinite reflection coefficient is that an inhomogeneous surface wave can exist at this frequency that is propagated parallel to the boundary but decays exponentially on either side of it. In 
our formulation the infinity arises because the wave can exist in the absence of an incident wave.

The point $\Omega=1$ is a branch point of $\chi$ and $\theta$ and hence of $R$ and $T$. The WKBJ solutions fail near this point and we should not, therefore, take the numerical results seriously near it. The corresponding formulae for $T$ are discontinuous at the branch point, but this is a consequence of the failure of the approximation.

Now consider the region $\Omega>1$. This is the situation considered by Walker (1998) who plotted $R$ and $T$ against $\Theta$ rather than $\Omega$. The amplitude of the reflection coefficient is unity showing that there is always total reflection, as must be the case, since there is no loss of energy between incident and reflected wave when the effect of the resonance is excluded. The phase, however, shows a series of changes of $2 \pi$ at each of which the phase changes rapidly with frequency. These are coincident with a series of peaks in the amplitude of $T$ occurring where $\Theta=n \pi$. Near these frequencies the signal in the guide is strong: at other frequencies not much of the signal penetrates the magnetopause. Walker (1998), used Rayleigh's method of stationary phase to discuss the nature of the signal. If we assume that the incident signal can be represented by a Fourier transform of the form

$f_{I}(x, t)=\int_{-\infty}^{\infty} A(\omega) e^{-i\left\{\omega t-\int_{0}^{x} k_{x}(\omega) d x\right\}} d \omega$

with a factor $\exp \left\{i\left(k_{y} y+k_{z} z\right)\right\}$ suppressed, then the reflected signal has the form

$f_{R}(x, t)=\int_{-\infty}^{\infty} A(\omega) R(\omega) e^{-i\left\{\omega t+\int_{0}^{x} k_{x}(\omega) d x\right\}} d \omega$.

The method of stationary phase uses the assumption that the only significant contribution to the signal arises where there is constructive interference between the plane wave Fourier components. This occurs where the phase of the integrand is stationary with respect to changes in $\omega$. At the boundary, where $x=0$, this occurs for the incident wave where

$\frac{\partial}{\partial \omega}\{\omega t-\operatorname{pha}(A)\}=t-\frac{d}{d \omega}[\operatorname{pha}(A)]=0$

and for the reflected wave where

$$
\begin{aligned}
& \frac{\partial}{\partial \omega}\{\omega t-\operatorname{pha}(A)-\operatorname{pha}(R)\} \\
& =t-\frac{d}{d \omega}[\operatorname{pha}(A)+\operatorname{pha}(R)] \\
& =0 .
\end{aligned}
$$

This implies that each frequency component in the reflected spectrum suffers a delay

$\tau=\frac{d}{d \omega}[\operatorname{pha}(R)]$

relative to the incident wave. If we examine the dependence of the phase of the reflection coefficient on $\Omega$ we see that near a series of discrete frequencies it has a steep slope. At these frequencies the delay is large: at other frequencies it is small.
Walker (1998) interprets this as the capture of the wave in the guide at frequencies corresponding to the waveguide modes. At these frequencies there are peaks in the transmission coefficient showing a larger amplitude of signal in the guide. The process may be visualised as one in which most frequencies are reflected at the boundary with small transmission into the guide. Near the resonant frequencies, however, the small amount of the wave that is transmitted undergoes a phase change of $2 n \pi$ as it is reflected back and forth across the guide. After each reflection it is in phase with the incident wave and there is constructive interference, building up a signal in the guide. After some time the captured frequencies begin to get out of phase, and eventually after time $\tau$ the frequency component leaks from the guide.

In what follows we discuss how this picture of cavity excitation is modified by the presence of the resonance and by the velocity of the magnetosheath plasma.

\subsection{Coupling to the resonance}

The treatment above assumes that the variation of the Alfvén speed beyond the turning point is so slow that coupling to the resonance can be ignored. With the model used this is not, in fact, so. The reflection at the turning point is not perfect. Walker (2000b) computes the transmission to the resonance. In Fig. 3 we show a computation of the fields as a function of $\zeta$, using his method, for typical resonance conditions. The top panel shows the generalised pressure $\psi=p+\mathbf{B} . \mathbf{b} / \mu_{0}$, the second the displacement $\xi$ in the $x$ direction, and the third the transverse displacement $\eta$ in the $y$ direction. The turning point is at $\zeta=0$ and the resonance at $\zeta=0.5$. The magnetopause is near one of the nodes of $\xi$. The pressure $\psi$ is continuous across the resonance, the poloidal displacement $\xi$ has a logarithmic singularity, and the resonant transverse Alfvén wave $\eta$ has a pole at the resonance. The Stokes constant $S$, representing the reflection coefficient at the turning point, is not $-i$ but must be found by other means. Walker (2000b) has shown how this constant may be determined numerically. In Fig. 4 it is plotted as a function of $\zeta_{R}=\left(k_{y} x_{R}\right)^{2 / 3}$, the normalised distance between the turning point and resonance.

In the absence of coupling to the resonance the amplitude of the Stokes constant is unity and the phase $-90^{\circ}$. When the resonance effect is included then, when $k_{y}=0$ and hence $\zeta_{R}=0$, there is no coupling and the Stokes constant is unchanged as $-i$. As $\zeta_{R}$ increases, the amplitude and phase of $S$ decrease. The amplitude is minimum at about $\zeta_{R}=0.4$ and increases to unity as $\zeta \rightarrow \infty$. The phase continues to decrease approaching $-270^{\circ}\left(+90^{\circ}\right)$ as $\zeta \rightarrow \infty$. Since the Stokes constant represents a reflection coefficient, defined as the ratio of the incident to the reflected WKBJ solution, this shows that there is a value of $\zeta_{R}$ and hence of $k_{y}$ for which reflection is minimum and transmission to the resonance a maximum. For typical models this value of $k_{y}$ yields values of azimuthal wave number $m \simeq 3$ or 4 (Walker, 2000b).

Figure 5 shows the reflection and transmission coefficients when the Stokes constant is calculated in this way. The model is otherwise exactly the same as in Fig. 2. For $\Omega<1$ 

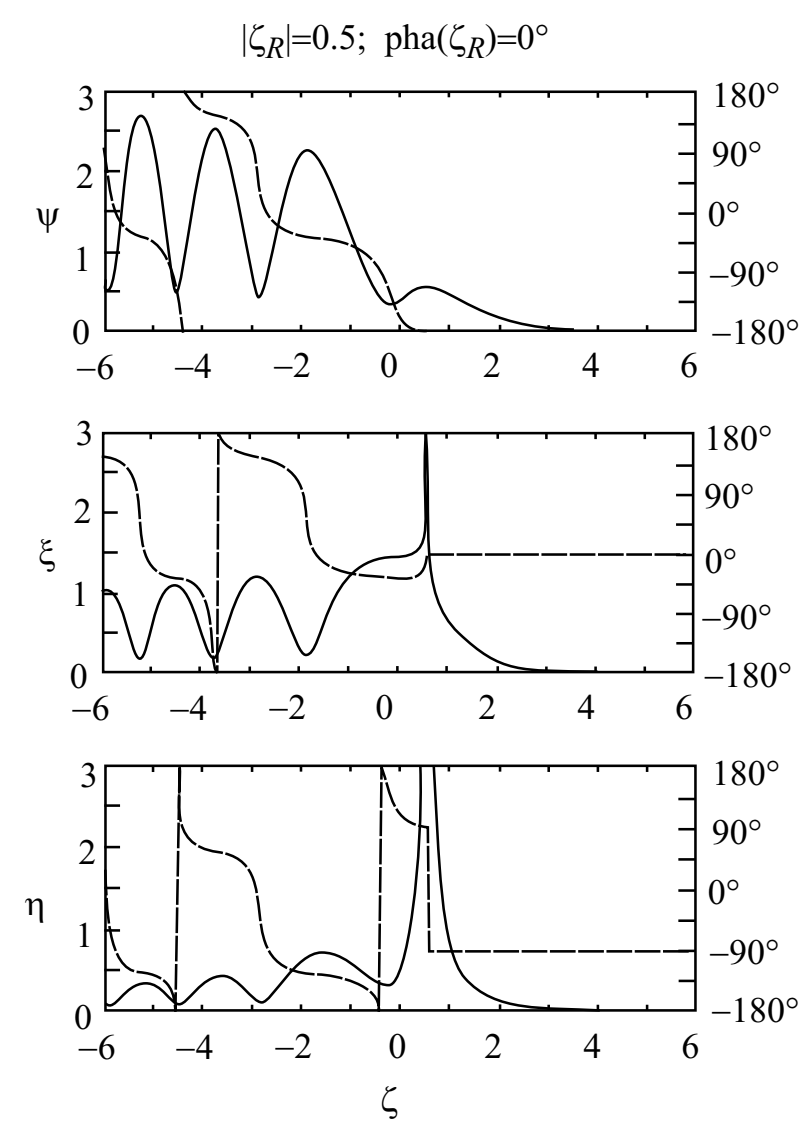

Fig. 3. Computation of fields in the neighbourhood of a turning point and a field line resonance (see text for explanation)
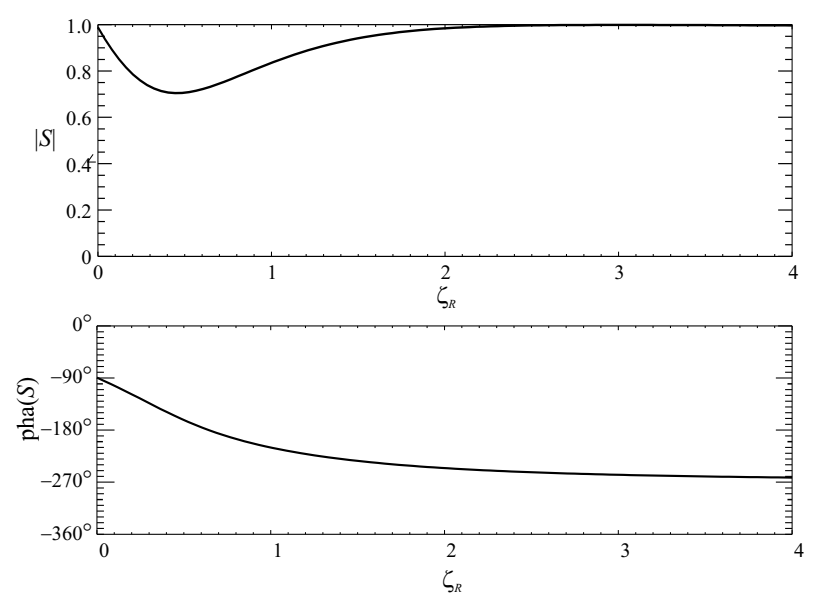

Fig. 4. Stokes constant when the effect of the resonance is included. No losses: $\Im\left(\zeta_{R}\right)=0$.

the behaviour is given by Eqs. (14) and (15) as is the case in Fig. 2. We concentrate on the behaviour for $\Omega>1$.

The modulus of the reflection coefficient shows a series of minima at frequencies corresponding to the positions of the waveguide modes. Between these frequencies it rises almost
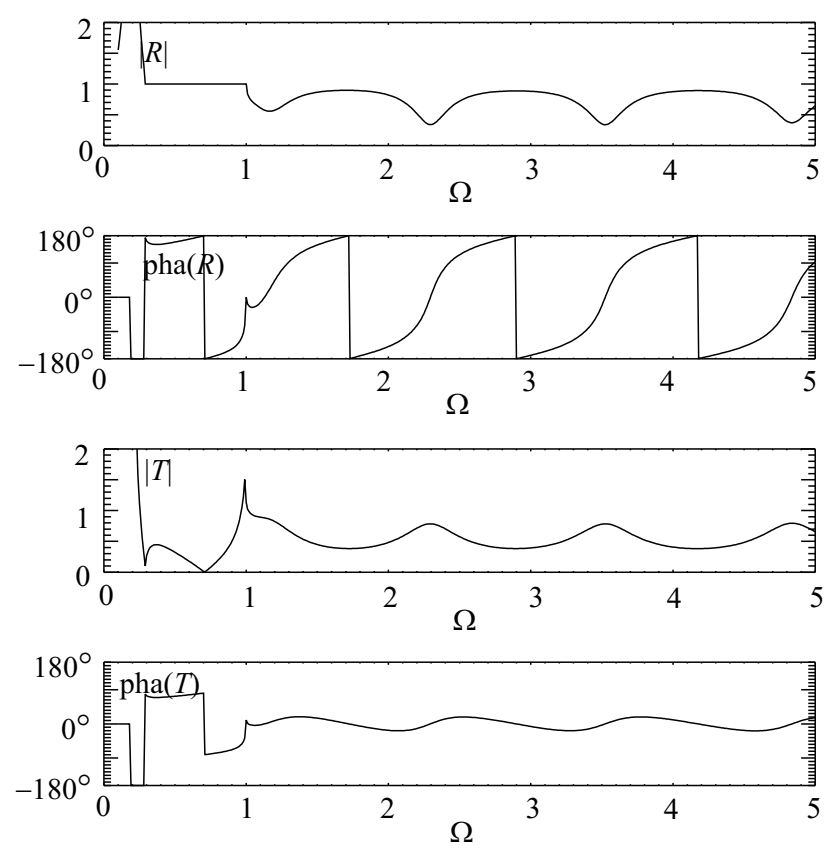

Fig. 5. Amplitude and phase of the reflection and transmission coefficients when the effect of the resonance is included. $\rho_{0,2}=0.1 \rho_{0,1}$, $k_{z}=3 / a L_{0}, k_{y}=3 / a L_{0}, V / V_{A, 0}=0$.

to unity. The phase still has a steep slope near the modes. The amplitude of the transmission coefficient has a similar form to the case when the resonance is neglected but the peaks are much less pronounced.

The interpretation of this is that, since not all the energy is reflected at the turning point, the reflection coefficient at the boundary must always be less than unity. Further, there can only be efficient coupling to the resonance when a significant fraction of the energy is captured in the cavity. The peaks in the transmission amplitude occur at the waveguide modes. It is debatable whether the waveguide analogy is most appropriate in such circumstances. The situation is more analogous to a thin film coating on a lens, allowing for maximum transmission at critical frequencies. Indeed, as described by Walker (2002), the distance of the turning point from the magnetopause varies with longitude. Rather than a uniform thin film the cavity has a geometry more like an optical wedge increasing in thickness with distance from noon. Thus the frequencies of maximum transmission, corresponding to the waveguide modes, are functions of longitude. In such circumstances it is difficult to see how oscillations with frequencies determined by the cavity can drive field line resonances at precise frequencies extending over many hours of local time as observed, for example, by Walker et al. (1992) or Fenrich et al. (1995).

\subsection{Moving magnetosheath}

Now let us suppose that the magnetopause is moving relative to the magnetosphere. In Fig. 6 we show the reflection 

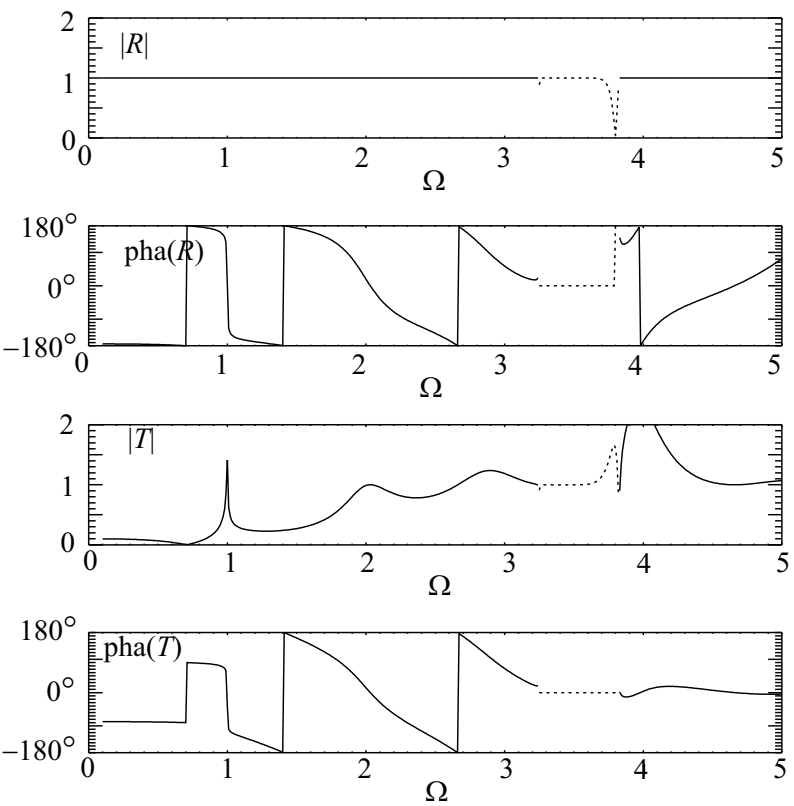

Fig. 6. Amplitude and phase of the reflection and transmission coefficients when excitation of the resonance is negligible. $\rho_{0,2}=0.1 \rho_{0,1}, k_{z}=3 / a L_{0}, k_{y}=3 / a L_{0}, V / V_{A, 0}=5$.

and transmission coefficients on the real axis when the normalised streaming velocity is $V / V_{A, 0}=5$. If this curve is compared with Fig. 2 there are a number of significant differences. Note that the curve is shown dotted where $3.24<\Omega<3.83$. In this region $k_{x}^{2}$ is negative in the magnetosheath because

$\omega_{0}^{2} \equiv\left(\omega-k_{y} V\right)^{2}<\left(k_{y}^{2}+k_{z}^{2}\right) V_{S}^{2}$.

The incident and reflected waves are therefore evanescent. As described above, this does not necessarily mean that no energy can reach the guiding region from outside; if there is a nearby magnetosheath region where propagation is possible then barrier penetration may occur.

Examination of the behaviour in the region $1<\Omega<3.24$ shows that a naive application of the method of stationary phase leads to difficulties. The slope of the phase of the reflection coefficient is negative. Equation (36) would then imply that the wave packet would emerge from the cavity before the incident wave packet had arrived: there is clearly a causality problem. The reason is that we have not correctly chosen the contour for evaluating the integral (33). The two integrals (32) and (33) can be found by Laplace transforming the appropriate wave equation with a complex Laplace transform variable $i \omega$. The Laplace formalism requires that the integration must then be along a contour that lies above all the singularities in the integrand. A more rigorous description of the method of stationary phase (Budden, 1961, §15.19) requires that this contour be distorted to pass through the saddle points of the integrand. These generally occur at complex values of $\omega$. The phase is stationary at the saddle points. In the case considered above, where there is no coupling to the

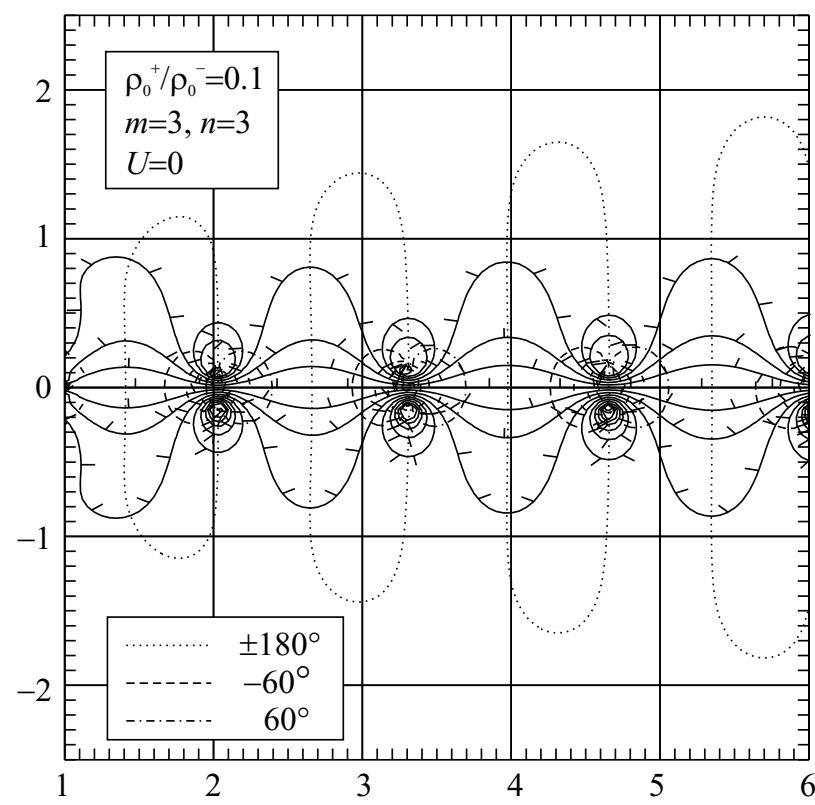

Fig. 7. Reflection coefficient contours when the magnetosheath plasma is at rest. The full contours represent amplitude with the ticks showing the downhill direction. The dotted lines are contours of constant phase. The amplitude contours are drawn for $|R|=10^{0.1 n}$ where $n=0, \pm 1, \pm 2, \pm 3, \ldots$. The real axis coincides with the contour $|R|=1$. There is a row of poles below the real axis and a corresponding row of zeros above it. The contours of constant phase are shown at $30^{\circ}$ intervals. the contours for pha $(R)=0, \pm 30^{\circ}$ are crowded between the poles and zeros.

resonance, these saddle points lie on the real axis and our argument is valid. When the resonance is taken into account they are close to the real axis and the rate of change of phase in the direction of the imaginary axis is small. To illustrate this Fig. 7 shows a plot of the reflection coefficient as a function of complex $\Omega$ when there is no magnetopause motion. It can immediately be seen that the rapid variation in phase on the real axis occurs as the real axis passes the series of poles lying just below it. The integrand of the Laplace integral in Eq. (33) is

$I=A(\omega) R(\omega) e^{-i\left\{\omega t+\int_{0}^{x} k_{x}(\omega) d x\right\}}$.

In addition to any singularities of $A(\omega)$ associated with the incident wave, it has poles where $R$ has poles. Since these are below the real axis the Laplace contour can be taken to coincide with the real axis.

Now consider the complex reflection coefficient shown in Fig. 8 for the conditions corresponding to Fig. 6. First we note that there are branch points where $k_{x}^{2}=0$ in the magnetosheath. A branch cut must emerge from each. We choose this to lie on the real axis joining the two points where (37) holds. It is represented by a heavy dotted line in the diagram. To the left of this in the diagram there are two pairs of poles and zeros. These poles now lie above the real axis. The Laplace contour cannot coincide with the real axis; it must pass above the poles. The poles separate the contour 

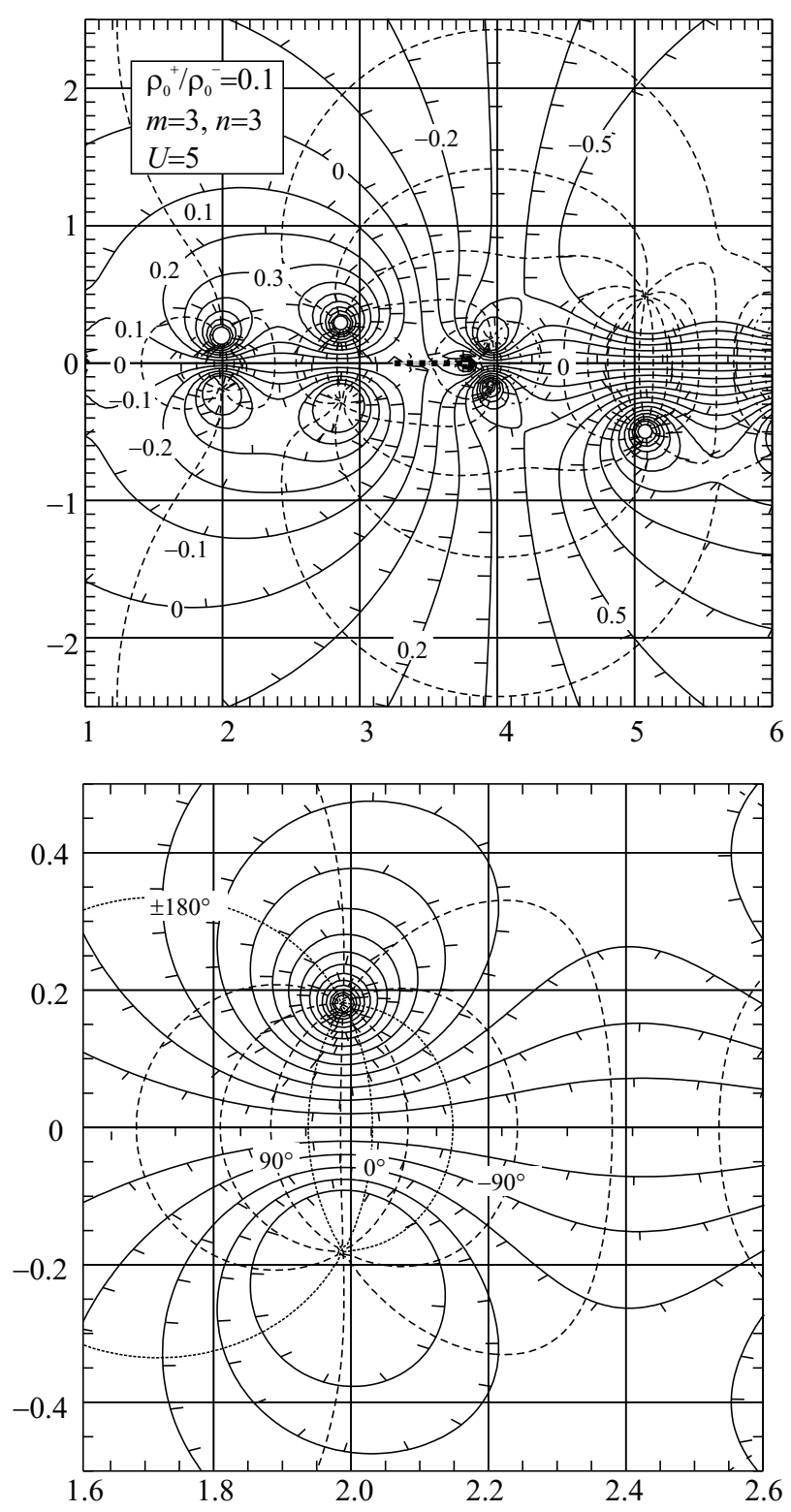

Fig. 8. Reflection coefficient in the complex plane when the magnetopause plasma is in motion. The lower panel shows the detail of a portion of the upper panel. The full contours represent amplitude with the ticks showing the downhill direction. The contours are drawn for $\log |R|=0.1 n$ where $n=0, \pm 1, \pm 2, \pm 3, \ldots$. The heavy dotted line is a branch cut. The dashed lines are contours of constant phase drawn at $30^{\circ}$ intervals.

from the real axis and the saddle points of the integrand are above them. We can either evaluate the integral by distorting the contour downwards and finding the residues at the poles as well as the value of the integral round the branch cut, or we can apply the method of stationary phase more rigorously by distorting it to pass through the saddle points of the integrand. The phase of $R$ varies along the contour through the saddle points in the correct sense for causality.

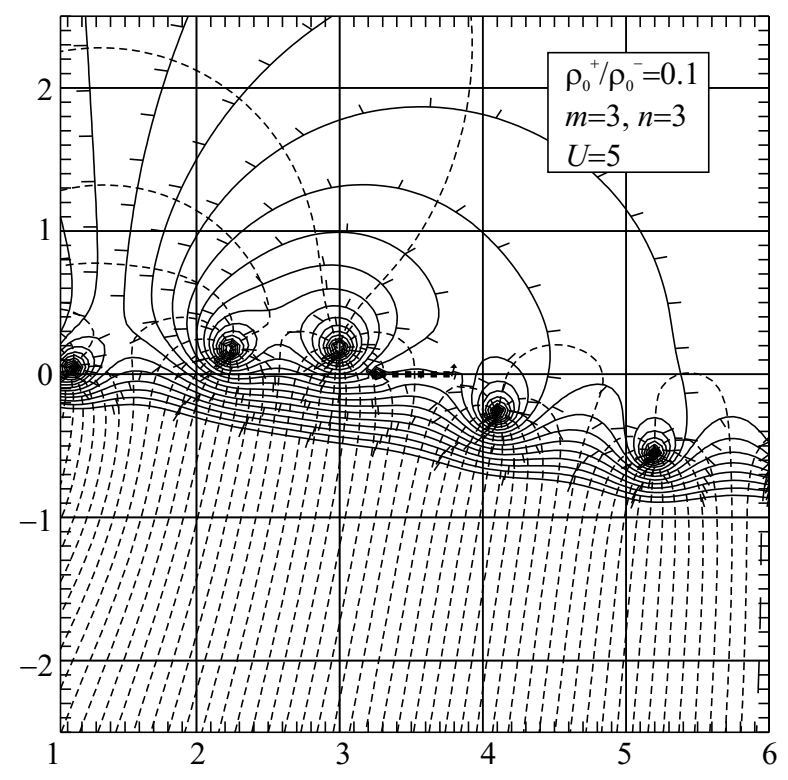

Fig. 9. Transmission coefficient in the complex plane when the effect of the resonance is taken into account and the magnetosheath plasma is in motion. The full contours represent amplitude with the ticks showing the downhill direction. The contours are drawn for $\log |R|=0.1 n$ where $n=0, \pm 1, \pm 2, \pm 3, \ldots$. The heavy dotted line is a branch cut. The dashed lines are contours of constant phase drawn at $30^{\circ}$ intervals.

Immediately on the other side of the boundary, inside the waveguide, in the same way as for reflection (33), the integral representation of the transmitted signal is of the form

$f_{T}(x, t)=\int_{C} A(\omega) T(\omega) e^{-\left\{\omega t-k_{x}(\omega) d x\right\}} d \omega$

where now $k_{x}$ is found from the dispersion relation within the magnetosphere. Again the contour $C$ must run above any singularities of the integrand. To illustrate the nature of the singularities that can arise we show in Fig. 9 the behaviour of $T$ in the complex plane when the magnetopause is in motion. In this case we have chosen the situation when losses to the resonance are taken into account. The transmission coefficient has the same set of poles as the reflection coefficient, since the denominator in the expressions for these quantities is the same. There are, however, no zeros in the region shown. The position of the poles is close to the position of those for the reflection coefficient when the resonance is ignored. They are, however, slightly closer to the real axis, so that the imaginary part of $T$ is smaller when there is leakage to the resonance.

\section{Illustrative examples}

\subsection{Integral representation}

Until now we have assumed that the incident signal varies harmonically in the $y$ and $z$ directions. A proper treatment of 
the problem would require a Laplace transform of the wave equations in time and Fourier transforms with respect to $x$ and $y$ to give an angular spectrum representation of the signal of the form

$g(x, y, z, t)=\iiint A e^{-i\left\{\omega t-k_{y} y-k_{z} z-k_{x}\left(\omega, k_{y}, k_{z}\right) x\right\}} d y d z d \omega(40)$

where $A$ is a function of $y, z, t$, the integrals with respect to $y$ and $z$ are along the real axis between limits $-\infty$ and $\infty$, and the integral with respect to $\omega$ is along the Laplace contour. For the purposes of our example suppose that the incident signal is a plane wave with direction determined by $k_{y}$ and $k_{z}$. It is switched on at an instant in time and varies sinusoidally thereafter with frequency $f$. Its intersection with the boundary plane $x=0$ is of the form

$g_{i}(y, z, t) \equiv H\left(t^{\prime}\right) \sin 2 \pi f t^{\prime}$

where

$t^{\prime}=t-\frac{k_{y} y+k_{z} z}{2 \pi f}$

and $H\left(t^{\prime}\right)$ is the Heaviside unit step function. The origin of time is chosen so that the first disturbance reaches the boundary point $y=0, z=0$ at $t=0$. The dependence on $x$ is of the form $\exp \left\{+i k_{x}\left(f, k_{y}, k_{z}\right) x\right\}$ for $x<0$.

We can perform a Laplace transform with respect to $t^{\prime}$ to obtain an integral representation of the form

$g_{i}(y, z, t)=\frac{1}{2 \pi} \int_{C} \frac{e^{-i \omega\left\{t-\left(k_{y} y+k_{z} z\right) / 2 \pi f\right\}}}{\omega^{2}-4 \pi^{2} f^{2}} d \omega$

The reflected signal is then

$g_{r}(y, z, t)=\int_{C} \frac{R\left(\omega, k_{y}, k_{z}\right) e^{-i \omega\left\{t-\left(k_{y} y+k_{z} z\right) / 2 \pi f\right\}}}{2 \pi\left(\omega^{2}-4 \pi^{2} f^{2}\right)} d \omega$

with dependence on $x$ of the form $\exp \left\{-i k_{x}\left(f, k_{y}, k_{z}\right) x\right\}$ for $x<0$.

4.2 Evaluation of the integral when the magnetosheath velocity is zero

Consider the evaluation of the integral (44) at the position on the boundary where $y=z=0$. It has poles where $\omega= \pm 2 \pi f$ and where the reflection coefficient has poles. For causality the contour $C$ must pass above all the singularities. When $t<0$ the contour can be closed in the upper half plane. No singularities are enclosed and the result is zero. When $t>0$ the contour can be distorted downwards as illustrated in Fig. 10. Because of the exponential factor the integral along the portion of the contour parallel to the real axis is negligible when $\Im(\omega)$ is negative and sufficiently large in magnitude. The portions parallel to the imaginary axis cancel and the integral is given by the sum of the residues at the poles and any branch cut integrals associated with the reflection coefficient. We concentrate on the contribution from the poles at $\omega= \pm 2 \pi f$ and one of the pairs of poles associated with the reflection coefficient, assumed to be located at $\omega= \pm \omega_{R}-i \gamma$,

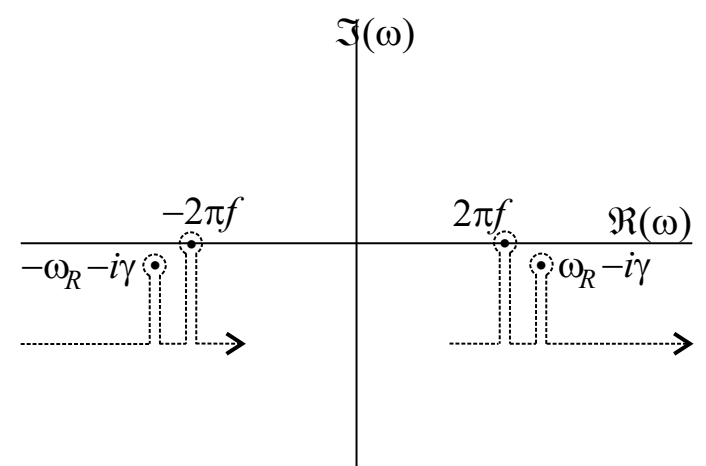

Fig. 10. Distortion of contour.

which is near the pole at $2 \pi f$. The symmetry about $\Re(\omega)=0$ ensures that the integral is a real function in the time domain so we need only concentrate on the poles with $\Re(\omega)>0$.

Let

$R(\omega)=\frac{G(\omega)}{\omega-\left(\omega_{R}-i \gamma\right)}$

The contribution to the integral of the poles under consideration is then $-2 \pi i$ times the sum of the residues, the negative sign arising because the contour passes clockwise round the poles. The contribution from other singularities is smaller because of the factor $1 /\left(\omega^{2}-4 \pi^{2} f^{2}\right)$ which is small when the singularities are far from $2 \pi f$. These contributions will be neglected.

With these assumptions, for $t>0$,

$$
\begin{aligned}
& g_{r}(0,0, t) \\
& \simeq-i \frac{\left.G(2 \pi f) e^{-2 \pi i f t}-G\left(\omega_{R}-i \gamma\right) e^{-\gamma t} e^{-i \omega_{R} t}\right)}{2 \pi f-\left(\omega_{R}-i \gamma\right)}
\end{aligned}
$$

When the two poles are close to each other we can write

$$
G\left(\omega_{R}-i \gamma\right) \simeq G(2 \pi f)+\left(2 \pi f-\omega_{R}+i \gamma\right) G^{\prime}(2 \pi f)
$$

and thus, by using (45), we may write

$$
\begin{aligned}
g_{r}(0,0, t) \simeq & -i R(2 \pi f)\left\{e^{-2 \pi i f t}-e^{-\gamma t} e^{-i \omega_{R} t}\right\} \\
& +i G^{\prime}(2 \pi f) e^{-\gamma t} e^{-i \omega_{R} t} .
\end{aligned}
$$

When the corresponding results for negative frequencies are included the result is real; the behaviour in the time domain is given by the real part of this expression.

Figure 11 shows the result of this calculation when $f=\omega_{R} / 2 \pi$. It is assumed that $\left|G^{\prime}\right| \ll|R|$ and this contribution is neglected. At this resonance frequency $\Theta=n \pi$ and $R$ is real and equal to unity so that the real part of the expression gives a sinusoidal behaviour. The arrival of the wave packet at $t=0$ sets up a forced reflected oscillation (top panel) associated with the pole at $\omega=2 \pi f$. It also excites a waveguide mode, oscillating at the same frequency and decaying because of leakage through the magnetopause. The leaked signal contributes to the reflected wave as shown in the middle panel. It is $180^{\circ}$ out of phase with the forced reflected 


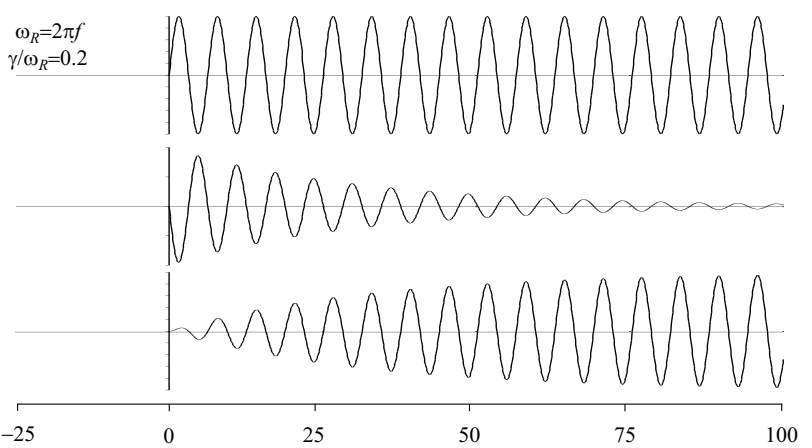

Fig. 11. Top panel: Directly driven reflected signal when $\omega_{R}=2 \pi f$. Middle panel: Contribution from excited leaky waveguide mode. Bottom panel: Superposition.

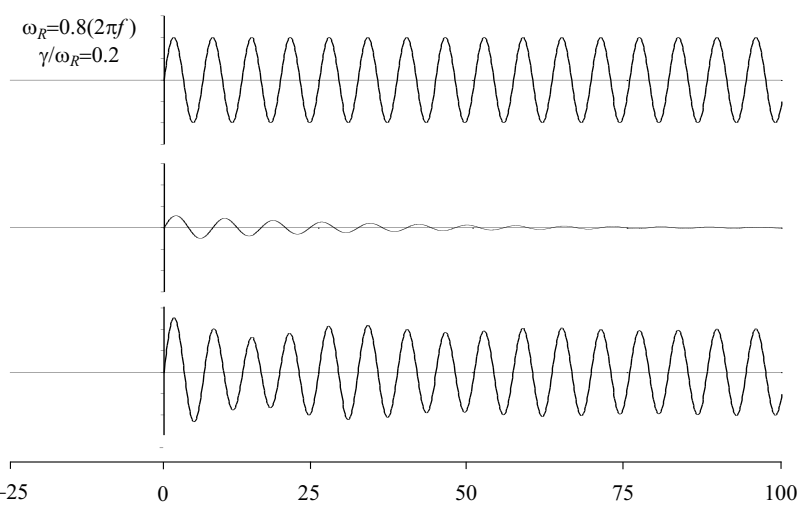

Fig. 12. Top panel: Directly driven reflected signal when $\omega_{R}=1.6 \pi f$. Middle panel: Contribution from excited leaky waveguide mode. Bottom panel: Superposition.

wave and initially cancels it. The superposition of the two waves gives the behaviour in the bottom panel. It shows that the reflected wave is initially zero and only grows to its final steady state value after a significant time delay. This is a more accurate description of the time delay predicted by the method of stationary phase (Walker, 1998).

When the pole associated with the driving frequency is well removed from the waveguide frequency then, for $t>0$, Eq. (46) may be written

$$
\begin{aligned}
& g_{r}(0,0, t) \\
& \simeq-i R(2 \pi f) e^{-2 \pi i f t}+i \frac{G\left(\omega_{R}-i \gamma\right) e^{-\gamma t} e^{-i \omega_{R} t}}{2 \pi f-\left(\omega_{R}-i \gamma\right)}
\end{aligned}
$$

The first term is of the same magnitude, since $|R|=1$. Its phase, however, is not zero, as shown in Fig. 2. It varies rapidly as distance from the resonance increases, approaching the value $\pm 180^{\circ}$. The second term, representing the resonance, is smaller in amplitude since $2 \pi f \neq \omega_{R}$ and the denominator is therefore larger in magnitude. Its frequency is also different. As a consequence the two contributions rapidly lose phase coherence as the waveguide mode decays. The resultant reflected signal rapidly rises to its final value.
This is associated with the fact that the guide is only weakly excited. The mismatch ensures that the wave is essentially reflected from the magnetopause without entering the magnetosphere. An illustration of such a case is shown in Fig. 12.

So far we have only considered the reflected signal. Let us now examine the nature of the transmitted signal in the guide. The total signal in the guide at $x=0$ is given by

$g_{t}(y, z, t)=\int_{C} \frac{T\left(\omega, k_{y}, k_{z}\right) e^{-i \omega\left\{t-\left(k_{y} y+k_{z} z\right) / 2 \pi f\right\}}}{2 \pi\left(\omega^{2}-4 \pi^{2} f^{2}\right)} d \omega$

This can be considered in exactly the same way as for the reflected wave. The general characteristics of the solution are the same. However, unlike the reflection coefficient, the transmission coefficient does not have a constant unit amplitude. The maximum value of its amplitude is unity at a frequency that matches the real part of the frequency of the wave guide mode. Here the transmitted signal just inside the magnetopause behaves in much the same way as the reflection coefficient as shown in Fig. 11. Throughout the guide the dependence on $x$ is of the form

$\cos \left\{\int_{0}^{x} k_{x} d x\right\}$

When the frequency is not near the frequency of a characteristic mode the qualitative behaviour is similar to that of the reflection coefficient shown in Fig. 12 but the amplitude is much smaller because the amplitude of the transmission coefficient is significantly less than unity. The signal in the guide rises until it reaches a steady driven value.

The effect of allowing for leakage to the resonance is to increase the losses. Energy is fed steadily to the resonance.

4.3 Evaluation of the integral when the magnetosheath velocity is non-zero

When the magnetosheath velocity is large enough, so that $\omega<k_{y} V$, we have seen that the poles of the reflection and transmission coefficients may lie above the real axis as shown in the examples of Figures 8 or 9 . In this case, when the residue at the pole is evaluated, it has a factor $e^{+\gamma t}$. Initially, depending on the value of the driving frequency, the solution for reflected or transmitted wave is of similar form to (48) or (49) with $-\gamma$ replaced by $+\gamma$. The signal inside and outside the guide is unstable and initially grows. This is the same situation as described by Mann et al. (1999) except that there is a continuous driver. Even if the driver is switched off the signal continues to grow.

Of course, as with any instability the growth cannot continue without limit. The assumption of linearity is soon violated and the treatment breaks down. We expect this to modify the behaviour at the magnetopause. Non-linear behaviour will lead to turbulence and the broadening of the boundary to reduce the velocity gradient until the growth ceases. The effect of the resonance is to introduce additional losses, reducing the growth rate. 


\section{Discussion and Conclusions}

This paper discusses the way in which an external disturbance, which could be either a quasi-harmonic wave or a broad band signal, could excite magnetospheric oscillations and hence field line resonances. How relevant are its results to the observations?

An idealised, uniform, stable, mean magnetosphere has a variety of characteristic modes of oscillation. The frequencies of these are determined by the shape and dimensions of the structure and by the characteristic velocities of the plasma. The real magnetosphere is nonuniform and constantly changing in time. Any estimate of its natural frequencies of oscillation suggests that these too should be constantly changing. It is puzzling that discrete, stable oscillations of this system, with well defined frequencies, are regularly observed.

One class of normal modes, however, provides a continuum of frequencies. This is the toroidal oscillation: in a cylindrically symmetric geometry each $L$-shell has its own natural frequency of toroidal oscillation determined by the length of the field line and the density of the plasma distributed along it. It is important to appreciate that the observational evidence for the existence of discrete Pc5 oscillations with relatively small azimuthal wave number $m$ is based overwhelmingly on observations of field line resonances, which are toroidal oscillations at discrete latitudes resonating at their natural frequencies in response to an unobserved driver. (We exclude the classes of pulsations with large $m$ probably arising from drift-bounce resonance with energetic particles.) Only recently have coordinated observations (Mann et al., 2002) provided some direct evidence of field line resonances associated with waveguide modes and magnetopause oscillations. While the authors attribute these oscillations to Kelvin-Helmholtz instability on the magnetopause, there is no direct evidence of this. Magnetopause oscillations are the inevitable consequence of any source mechanism that sets the magnetospheric waveguide into oscillation.

What then are the possible source mechanisms that could initiate or sustain narrow band field line resonant oscillations? We discuss possibilities in turn:

1. Kelvin-Helmholtz instability: The Kelvin-Helmholtz instability, in its original sense, occurs when, at the magnetopause, $\omega^{2}<k_{y}^{2}+k_{z}^{2}$. This corresponds to $\Omega<1$. The instability is a surface wave, decaying exponentially with $x$ on either side of the boundary. For a sharp boundary the growth rate increases monotonically with increasing $\sqrt{k_{y}^{2}+k_{z}^{2}}$. It is necessary to allow for a boundary of finite thickness to quench this growth for shorter wavelengths (Walker, 1981). This determines a frequency of maximum growth which could give rise to an oscillation with well-defined frequency. Because of the evanescent decay such an instability can only excite a field line resonance that occurs at frequencies low enough for the resonant field line to lie close to the magnetopause. It can therefore explain the lowest frequency oscillations but cannot explain multiple resonances. Resonances at frequencies larger than 1 or $2 \mathrm{mHz}$ occur on field lines far from the magnetopause and are unlikely to be excited by a wave decaying exponentially with distance from the boundary. The more general Kelvin-Helmholtz instability described by Mann et al. (1999) occurs in conjunction with the a cavity. We deal with it in our next point.

2. Excitation of cavity at the boundary: Mann et al. (1999) examined the case where magnetosheath and magnetosphere were both uniform and in relative motion. They excluded the effect of resonance and replaced the reflection at the turning point by a reflection at a perfectly reflecting boundary. In the magnetosheath they applied the boundary condition that either the wave in the magnetosheath must be propagated away from the magnetopause or it must decay evanescently with distance from the magnetopause. While this is a substantial idealisation, their model can deal qualitatively with the effect of wave amplification at the boundary when a cavity with characteristic frequencies of oscillation is present. Because of the boundary condition applied in the magnetosheath, it is limited to a waves generated by instability or over-reflection at the magnetopause. They find that such waves occur when the velocity of the magnetosheath plasma exceeds a certain value. Such a mechanism can explain the occurrence of pulsations at times when the solar wind is sufficiently large. If the effect of the resonance had been included it would have predicted the existence of multiple resonances at latitudes corresponding to the natural frequencies of toroidal oscillation. Such a mechanism does not explain the stability of the frequencies that are generated since the dimensions of the magnetospheric cavity are not constant, particularly at times of large solar wind speed. It also cannot explain the occurrence of pulsations when the solar wind speed is smaller. Mann et al. (1999) note that the condition for excitation of the modes correlates well with the observation that Pc5 power increases when the solar wind speed exceeds $500-600 \mathrm{~km} \mathrm{~s}^{-1}$. However, when solar wind speeds are less than this the mechanism is inoperative and cannot explain the existence of oscillations at times of lower solar activity.

3. External source: This paper examines the effect of an external source. The difference in principle with the work of Mann et al. (1999) is that there are different boundary conditions in the magnetosheath. The treatment allows for an incoming and and outgoing wave. Other differences are the allowance for a varying magnetosphere and for transmission to the resonance. In the illustrated example we have treated the case of an incident sinusoidal signal that grows from zero and is incident on the magnetopause at an angle determined by $k_{y}$ and $k_{z}$. The initial rise in the signal reaches the 
point $(0,0,0)$ on the magnetopause at time $t=0$. A similar treatment is, however, applicable to any incident wave form. Provided that there is incident power in the frequency band close to a normal mode that mode will be excited. What happens subsequently depends on the properties of the normal mode. For small magnetosheath speeds the normal modes decay because they leak from the cavity to the magnetosheath and to the resonance. The total signal in the guide is the sum of a forced oscillation and the normal modes. Initially the forced oscillation is cancelled by the normal mode. As the normal mode decays the cancellation becomes less and the total signal in the guide grows. Ultimately the only remaining signal is the forced oscillation and its amplitude depends on the amplitude of the transmission coefficient: if its frequency is close to the natural frequency the amplitude is large, if not it is small.

With these three possibilities in mind let us consider the nature of the pulsation source. All three scenarios may be operative in different conditions. In appropriate circumstances the simple Kelvin-Helmholtz instability (Walker, 1981) may be operative provided that the frequency is low enough for the wave to be evanescent at the magnetopause. If the solar wind speed, and hence the magnetosheath speed is sufficiently large then over-reflection at the magnetopause (Mann et al., 1999) may cause the normal modes to be amplified. This unstable situation does not require any external source - any small perturbation will trigger it. At lower speeds this mechanism does not operate because losses through leakage exceed the growth rate. In such circumstances an external source is necessary to provide the energy. This is the subject of the remainder of the discussion.

One possibility is a step function. In this case each mode is excited into oscillation with an amplitude determined by the power in the frequency band associated with the mode. The magnetosphere then rings like a bell, with each mode decaying as energy leaks out of the guide to the resonance and to the magnetosheath. Excitation of pulsations by pressure pulses has been observed by a number of authors (Mann et al., 1998; Prikryl et al., 1998; Korotova et al., 2000). Unless the solar wind speed is large enough to excite instability, the energy associated with each mode is just what was available in the associated frequency band of the driving pulse.

The other possibility is a harmonic driver in the solar wind as discussed in this paper. Recent observations (Stephenson and Walker, 2002; Kepko et al., 2002) have suggested that on some occasions the oscillations with discrete frequencies seen in the solar wind are correlated with observations of pulsations within the magnetosphere. The evidence is, as yet, sparse but suggests an interesting alternative for the source of some pulsations where the discrete frequencies are determined by the source rather than by the nature of the cavity. Then if the source provides a stable narrow band signal that is transmitted to the resonance through the less stable broader band filter provided by the cavity the stability of some observed field line resonances is explained.
The solar wind is usually thought of as turbulent. There is however compelling evidence of the existence of the existence of coherent waves occurring in the Pc5 band of frequencies. Thomson et al. (1995) have carried out an extended study of oscillations of the interplanetary magnetic field in both the microhertz and millihertz ranges and related these respectively to gravity $(g)$ waves and pressure $(p)$ waves in the helioseismic spectrum. The observations were made by the Ulysses spacecraft at distance of up to $5 \mathrm{AU}$ from the Sun. The analysis technique was a sophisticated multitaper method (Thomson, 1982) in which the data is analyzed using a succession of different windows designed to minimise spectral leakage from large lower frequencies to the smaller higher frequencies. At the same time it gives approximately equal weight to the whole time series, unlike a Hanning window, for example, which weights the data in the middle of the time series much more heavily. In particular Thomson et al. (1995) found strong correlation of the frequencies of the Pc5 IMF oscillations with those of the $p$ waves occurring at the Sun's surface. A variety of different statistical tests countered criticism (Roberts et al., 1996; Thomson et al., 1996) by showing that the likelihood of the null hypothesis that the correlations occurred by chance was very small. Although no direct link has been demonstrated, this raises the intriguing possibility that the frequencies of some Pc5 oscillations might be directly related to frequencies determined by a solar source.

This paper is not intended to provide a means of distinguishing between these sources which may all be operative in different circumstances. It is to be hoped that it may provide a tool for helping to constrain the interpretation of observations. Its chief results are:

1. The reflection coefficient for harmonic waves incident on the magnetopause from outside has been calculated as a function of frequency using a plane stratified model. The effects of leakage to the resonance and of a moving magnetosheath are included. Its properties are:

(a) When the magnetosheath speed is small and leakage to the resonance is neglected, for frequencies large enough for there to be a turning point within the magnetosphere, the magnitude of the reflection coefficient is unity. Its phase increases rapidly through $360^{\circ}$ near each waveguide mode frequency.

(b) When leakage to the resonance is included, and $k_{y}$ has a value close to that providing most efficient transfer to the resonance, the reflection coefficient amplitude is less than one but has minima near the mode frequencies representing energy that leaks to the resonance rather than being reflected.

(c) When the magnetopause speed is sufficiently large the sense in which the phase of the reflection coefficient changes is reversed for lower frequencies: it decreases rapidly through $360^{\circ}$ near these frequencies. 
2. The corresponding behaviour for the transmission coefficient into the waveguide is that the amplitude shows peaks near the mode frequencies.

(a) When transmission to the resonance is neglected and the magnetosheath speed is small the value of $T$ at the maxima is unity, showing that energy is efficiently transferred to the waveguide at these frequencies. At intermediate frequencies $T \simeq 0.4$ and much less energy is transferred to the guide. (It should be borne in mind that the energy content is proportional to $|T|^{2}$.)

(b) When the effect of the resonance is included the difference between maximum and minimum values of $T$ is much less. The discrimination between frequencies is not as strong.

(c) When the magnetosheath speed is sufficiently large the maximum value of $|T|$ may be greater than unity.

3. An incident wave packet can be represented as a modified Laplace integral with respect to frequency $\omega \equiv-i s$. For causality the contour of integration must run parallel to the real $\omega$ axis above any singularities. The reflected and transmitted signals can then be constructed by multiplying the integrand by $R$ and $T$.

(a) When the magnetosheath speed $V$ is less than $\omega / k_{y}$ the singularities lie below the real axis. The effect of the arrival of the wave packet is to excite the normal modes which interfere with the driven signal. Ultimately the modes decay and only the driven signal remains. The details depend on the form of the incident signal. It is important to note that there is substantial modification of the envelope of the reflected and transmitted signals. There are also many other such processes along the path of the signal so that one cannot expect to find excellent cycle by cycle correlation of oscillations in the solar wind with observed pulsations.

(b) When $V>\omega / k_{y}$ there are singularities above the real axis that correspond to growing modes that are excited by any small disturbance. It is not necessary to invoke an external source in such circumstances. The magnetopause boundary is unstable and will set the waveguide into oscillation.

The experimental determination of the source of field line resonances is not an easy task. Ideally one requires simultaneous solar wind data, magnetosheath data on the flanks and ground based data over an extended range of latitude and longitude. The crucial measurements needed are

- Spectral information from the ground based data establishing the existence of field line resonances as well as their frequency and azimuthal wavelength
- A determination of the speed of the magnetosheath plasma at the flanks of the magnetosphere near the longitude of the observed field line resonance. A determination of the magnetic field is also desirable.

- Solar wind velocity, magnetic field, density, and pressure (or equivalently temperature).

The first question is then whether multiple resonances can be seen at different latitudes. If so then a simple KelvinHelmholtz instability is not possible and one should look to an mechanism providing discrete frequencies. This could be through the excitation of cavity or waveguide modes or by incident harmonic waves originating from the solar wind.

Next one should enquire whether $\omega^{2}$ is greater or less than $k_{y}^{2} V^{2}$. If it is greater, then one can look to an instability on the magnetopause to excite the waveguide as described by Mann et al. (1999). If not one must look to an external source.

If an external source is indicated then one should look to a comparison of the solar wind data with the ground based data. Such a source may be impulsive or periodic. In either event, if there is no instability at the magnetopause, then the field line resonance must be directly driven. This requires that there be sufficient energy flux in the appropriate frequency band to supply the energy deposited in the ionosphere by the field line resonance. Estimates of this can be made from the available data (Stephenson and Walker, 2002).

Acknowledgements. The author is grateful for financial support from the South African National Research Foundation and the University of KwaZulu-Natal.

Topical Editor T. Pulkkinen thanks M. Ruohoiemi and another referee for their help in evaluating this paper.

\section{References}

Budden, K. G.: Radio waves in the ionosphere, Cambridge University Press, Cambridge, 1961.

Dungey, J. M.: Electrodynamics of the outer atmosphere, Penn State Ionosph. Res. Lab. Report 69; abridged version with same title in: The physics of the ionosphere, Phys. Soc. London, 29236, 1955.

Fenrich, F. R., Samson, J. C., Sofko, G., and Greenwald, R. A.: ULF high- $m$ and low- $m$ field line resonances observed with the super dual auroral radar network, J. Geophys. Res., 100, 21535 $21547,1995$.

Kepko, L., Spence, H. E., and Singer, H. J.: ULF Waves in the solarwind as direct drivers of magnetospheric pulsations, Geophys. Res. Lett., 29, 1197, doi:10.1029/2001GL014405, 2002.

Kivelson, M. G. and Southwood, D. J.: Resonant ULF waves: A new interpretation, Geophys. Res. Lett., 12, 49-52, 1985.

Kivelson, M. G., and Southwood, D. J.: Coupling of global magnetospheric eigenmodes to field line resonances, J. Geophys. Res. 91, 4345-4351, 1986.

Korotova, G. I., Kivelson, M. G., Sibeck, D. G., Potemra, T. A., and Staunings, P.: Multipoint observations of global magnetospheric compressions, J. Geophys. Res., 105, 23 293-23 302, 2000.

Mann, I. R., Chisham, G., and Bale, S. D.: Multisatellite and ground-based observations of a tailward propagating Pc5 mag- 
netospheric waveguide mode, J. Geophys. Res., 103, 4657-4669, 1998.

Mann, I. R., Wright, A. N., Mills, K. J., and Nakariakov, V. M.: Excitation of magnetospheric waveguide modes by magnetosheath flows, J. Geophys. Res., 104, 333-353, 1999.

Mann, I. R., Voronkov, I., Dunlop, M., Donovan, E., Yeoman, T. K., Milling, D. K., Wild, J., Kauristie, K., Amm, O., Bale, S. D., Balogh A., Viljanen A., and Opgenoorth, H. J.: Coordinated ground-based and Cluster observations of large amplitude global magnetospheric oscillations during a fast solar wind speed interval, Ann Geophys., 20, 405-426, 2002.

Prikryl, P., Greenwald, R. A., Sofko, G. J., Villain, J.-P., Ziesolleck, C. W. S., and Friis-Christensen, E.: Solar-wind-driven pulsed magnetic reconnection at the dayside magnetopause, Pc5 compressional oscillations, and field line resonances, J. Geophys. Res., 103, 17 307-17322, 1998.

Roberts D. A., Ogilvie K. W., and Goldstein M. L.: The nature of the solar wind, Nature 381, 31-32, 1996.

Ruohoniemi, J. M., Greenwald, R. A., Baker, K. B., and Samson, J. C.: HF radar observations of Pc5 field line resonances in the midnight/early morning MLT sector, J. Geophys. Res., 96, $15697-$ 157101991.

Samson, J. C., Harrold, B. G., Ruohoniemi, J. M., Greenwald, R. A., and Walker, A. D. M.: Field line resonances associated with MHD waveguides in the Earth's magnetosphere, Geophys. Res. Lett., 19, 441-444, 1992.

Southwood, D. J.: The hydromagnetic instability of the magnetospheric boundary, Planet. Space Sci., 16, 587-605, 1968.

Stephenson, J. and Walker, A. D. M.: HF radar observations of Pc5 ULF pulsations driven by the solar wind, Geophys. Res. Lett., 29, 1297, doi:10.1029/2001GL014291, 2002.

Thomson, D. J.: Spectrum estimation and harmonic analysis, IEEE Proc., 70, 1055-1096, 1982.

Thomson, D. J., Maclennan, C. G., and Lanzerotti, L. J.: Propagation of Solar oscillations through the interplanetary medium, Nature, 376, 139-144, 1995.
Thomson, D. J., Maclennan, C. G., and Lanzerotti, L. J.: The nature of the solar wind - reply, Nature, 381, 32-32, 1996.

Walker, A. D. M.: The Kelvin-Helmholtz instability in the low latitude boundary layer, Planet. Space Sci., 29, 1119-1133, 1981.

Walker, A. D. M.: Excitation of magnetohydrodynamic cavities in the magnetosphere, J. Atmos. Solar Terrest. Phys., 60, 12791293, 1998.

Walker, A. D. M.: Reflection and transmission at the boundary between two counterstreaming plasmas - Active boundaries or negative energy waves? J. Plasma Phys., 63, 203-219, 2000a.

Walker, A. D. M.: Coupling between waveguide modes and field line resonances, J. Atmos. Solar Terrest. Phys., 62, 799-813, 2000b.

Walker, A. D. M.: Excitation of Field Line Resonances by MHD Waves Originating in the Solar Wind, J. Geophys. Res., 107, 1481, doi:10.1029/2001JA009188, 2002.

Walker, A. D. M.: Magnetohydrodynamic waves in geospace, IOP Press Bristol, 2005.

Walker, A. D. M., Greenwald, R. A., Stuart, W. F., and Green, C. A.: Stare auroral radar observations of Pc5 geomagnetic pulsations, J. Geophys. Res., 84, 3373-3388, 1979.

Walker A. D. M., Ruohoniemi, J. M., Baker, K. B., Greenwald R. A., and Samson, J. C.: Spatial and temporal behavior of ULF pulsations observed by the Goose Bay HF radar, J. Geophys. Res., 97, 12 187-12 202, 1992.

Wright, A. N.: Dispersion and wave coupling in inhomogeneous MHD waveguides, J. Geophys. Res., 99, 159-167, 1994.

Wright, A. N. and Rickard, G. J.: ULF pulsations driven by magnetopause motions: Azimuthal phase characteristics, J. Geophys. Res., 100, 23, 703-723, 1995a.

Wright, A. N. and G. J. Rickard: A numerical study of resonant absorption in a magnetohydrodynamic cavity driven by a broad band spectrum, Astrophys. J., 444, 458-470, 1995 b. 